\title{
Persian At-Risk Women and Barriers to Receiving HIV Services in Drug Treatment: First Report From Iran
}

\author{
Mercedeh Samiei, ${ }^{1}$ Afsaneh Moradi, ${ }^{2}$ Roya Noori, ${ }^{3,}{ }^{,}$Sepideh Aryanfard, ${ }^{4}$ Hassan Rafiey, ${ }^{4}$ and Hooman \\ Naranjiha ${ }^{5}$ \\ ${ }^{1}$ Division of Psychiatry, Razi Psychiatric Hospital, University of Social Welfare and Rehabilitation Sciences, Tehran, IR Iran \\ ${ }^{2}$ Department of Psychology, Faculty of Psychology and Educational Sciences, Al-Zahra University, Tehran, IR Iran \\ ${ }^{3}$ Substance Abuse and Dependence Research Centre, University of Social Welfare and Rehabilitation Sciences, Tehran, IR Iran \\ ${ }^{4}$ Department of Social Welfare, University of Social Welfare and Rehabilitation Sciences, Tehran, IR Iran \\ ${ }^{5}$ Razi Drug Treatment Centre, Tehran, IR Iran \\ "Corresponding author: Roya Noori, Substance Abuse and Dependence Research Centre, University of Social Welfare and Rehabilitation Sciences, Tehran, IR Iran. Tel/Fax: \\ +98-2122180095, E-mail: roya1.noori22@gmail.com
}

Received 2015 February 03; Revised 2015 March 26; Accepted 2015 April 14.

\begin{abstract}
Background: As the most populated Persian Gulf country, in Iran, engagement with drug injection and unsafe sex are the main routes of HIV transmission among some drug-dependent women.

Objectives: The current study explored the barriers that a group of drug-dependent women reported in accessing and adhering to HIV services in drug use treatment.

Patients and Methods: Qualitative interviews were conducted with 47 Persian women at five outpatient drug treatment centers between January and December 2011. Five focus group interviews were conducted with ten key informants (KI). The interviews made use of a semi-structured interview guide, which facilitated discussion regarding the barriers. The interview transcripts were analyzed thematically using Atlas-ti software.

Results: The interview accounts highlighted a number of barriers, including stigmatization, a considerable lack of knowledge about free HIV centers in the community, previous traumatic events, misconceptions about the quality of HIV services, and a poor supportive referral system among drug treatment and HIV centers.

Conclusions: The findings highlight a need for removing stigma and providing high quality women-only HIV services. Increasing trust and knowledge of available HIV services are needed for this group of women. Increasing staff knowledge is a priority. An integrated supportive network among drug treatment and HIV centers is suggested in Iran.
\end{abstract}

Keywords: HIV, Women, Iran, Asia

\section{Background}

Iran (Persia), is a vast Persian-speaking country which is situated between the Caspian sea in the north and the Persian Gulf and the sea of Oman in the south. Red carpets, Persian Gulf, and the Persian Empire are the ancient symbols of Iran. With a population of 78 million, Iran is the most populated Persian Gulf country in western Asia.

Low quality opiates are the main illicit drugs in Iran. An estimated 2 - 3 million people are dependent on illicit drugs in Iran. Overall, six percent of them are women. Approximately, 200,000 people inject drugs in Iran (1). Among HIV-positive patients in Iran, $8.7 \%$ of them are women (2). Most of them have become HIV-infected by engagement with unsafe heterosexual relationship or injecting drugs (2).

Women are more likely than men to inject drugs, use drugs with partners, exchange sex for money or drugs, and have difficulties negotiating condom use (3). Therefore, this interplay of gender and risk behaviors creates an environment in which women are more at risk than men for acquiring HIV infection (4).

Recent studies in Iran found that at-risk women were more likely to misuse drugs and ignore condom use in sex (5-7). A study of 256 at-risk women in Tehran found that most of them had good HIV knowledge but ignored condom negotiation and had multiple sexual partners (8). These behaviors occur because at-risk women generally do not identify themselves as vulnerable to $\operatorname{HIV}(5)$.

In recent decades, Iran has widely implemented approved prevention and treatment programs to reduce HIV infection, such as antiviral therapy and methadone maintenance treatment (9). This is the only comprehensive drug and HIV treatment system in the region. Nevertheless, some at-risk women do not visit HIV centers because of their cultural misconceptions about HIV (6) or stigmatization (10). This issue can lead to a health concern due to 
the risk of HIV acquisition and subsequent transmission to others in the Persian community.

\section{Objectives}

Implementing successful HIV services in drug treatment should match the specific needs of women. To partly address this gap in literature, the current qualitative study aimed to explore the barriers to accessing and adhering to HIV services in drug treatment among a group of at-risk women.

\section{Patients and Methods}

\subsection{Study Design and Setting}

The study was conducted at five outpatient womenonly drug treatment centers between January and December 2011. All the centers were located in densely populated areas of Tehran. The study was approved and funded by the United Nations office on drugs and crime (UNODC) in Tehran.

We selected a qualitative methodology because of a dearth of information regarding barriers to accessing and adhering to HIV services among at-risk women. We also chose to conduct focus group discussions and qualitative interviews to generate insights into how HIV counseling and treatment support offerings were perceived or experienced by women (11).

\subsection{Participants and Sampling}

Participants $(\mathrm{n}=35)$ were mainly approached by welltrained staff members regarding their potential participation in the study. The staff members at each center had worked with at-risk women for at least five years. One quarter of the participants $(n=12)$ were recruited through research advertisements pinned to the notice board at each center. Participation was confidential and voluntary. All women were eligible to enter the study if they 1) were > 18 years, 2) were current drug treatment seekers, and 3) reported being at-risk within the past 12 months. Exclusion criteria included reporting severe drug withdrawal and/or intoxication symptoms, which were likely to interfere with conducting the interviews.

The word "at-risk" was defined as having a current HIVpositive status, reporting regular or temporary sex work, having or living with more than one sexual partner, having a HIV-positive spouse or man friend, being imprisoned, sharing needles and syringes, and/or engagement with drug injection within the past 12 months prior to recruitment.

\subsection{Study Procedure}

\subsubsection{Individual Interviews}

All participants provided informed written consent at the time of recruitment. An interview topic guide was used to facilitate and address a range of topics, including their needs and barriers to accessing and adhering to HIV services in drug treatment.

After the purpose of the study was explained, semistructured, in-depth interviews were conducted with each participant. These interviews were conducted by the second and third authors. Each interview took 60 - 90 minutes and was digitally recorded. A moderator noted any changes in body language that could have informed the analysis. Each interview was conducted at drug treatment centers, a research center, a cafe, or other places in their neighborhood to increase the convenience and comfort of the participants.

The participants received retail gifts for study participation. The interviews were transcribed verbatim by a research assistant and then reviewed by the interviewers for accuracy. The recordings were transcribed in full using a commercial transcription service and checked for accuracy before the analysis commenced.

\subsubsection{Focus Group Discussions (FGD)}

Five FGD were conducted with 10 key informants (KI). There were two to three people in each group. KI were from a range of governmental and private HIV and drug treatment centers. KI included the director of a HIV counseling and treatment center; an experienced field worker who worked with at-risk women; two doctors who were HIV specialists; a social worker and a clinical psychologist from the drug treatment centers; three experienced HIV service providers; and a registered nurse who had worked with atrisk women for 11 years.

Each FGD took 45 - 65 minutes and was digitally recorded. In addition, the moderator noted any changes in the group dynamic that might have added important information to the analysis. FGD were conducted to compare participant and KI perspectives on participant barriers, the quality and priorities of HIV services, and their suggestions for improving access and adherence to these services.

All FGD were facilitated by two of the study researchers. Each group provided a different perspective to deepen our understanding of their service utilization, needs, and deficiencies. Interviews with new women and KI continued until no new themes emerged in four consecutive interviews to assure data saturation (12).

\subsubsection{Data Analysis}

Data analysis focused on how the self-perceived barriers prevented at-risk women from accessing and adher- 
ing to HIV services in the community. The interview transcripts were imported into Atlas.ti qualitative software to assist with data management and coding. The software facilitated the management of the qualitative data, allowing our researchers to store, code, and retrieve texts, thus assisting in the deep identification of emerging themes rather than revealing new themes from the collected data.

We used a coding framework made up of the categories elicited from the interview topic guide to facilitate data analysis. Data were independently coded by two other team members (HR, HN) who met regularly with the interview team to discuss emerging themes and to revise the coding. Once the final categories were established, the interviewers reviewed the data to enhance their validity.

\subsubsection{Qualitative Data Quality}

There were some aspects of data quality that we applied to validate the collected data. Some of the strategies utilized included data triangulation, investigator triangulation, and methodological triangulation. Triangulation refers to the use of more than one approach to the investigation of a research question in order to increase confidence in the findings.

For data triangulation, we applied two methods of sample taking. As a result, the slices of data at different times and centers, as well as on the number of women, were gathered. For investigator triangulation, we hired more than one researcher in the field to gather and interpret the data. For methodological triangulation, we applied prolonged exposure in the field and member checks.

Through the use of prolonged exposure, we were able to form a rapport with the women and staff members and establish credibility as a temporary member of their group. The women's levels of comfort gradually increased as we spent more time in the field as participants and observers. Finally, by utilizing member checks with women and $\mathrm{KI}$, the data were further validated to ensure that we were accurately capturing their narratives.

These member checks with the women and KI provided authentication for their stories and personal experiences. The procedures that we undertook to guide the research and validate the experiences of women and KI were defined as reflexivity. Reflexivity refers to a researcher's work to critically monitor and understand the role of the self in the research endeavor (13). By utilizing these methods, we reduced the likelihood that the information collected or coded would be subject to researcher bias or systematic errors.

\section{Results}

\subsection{Sample Characteristics}

More than half of the women were opiate users, while the remaining women were co-users of impure opiates with low quality methamphetamine. The median age of the sample was 33 years. The median years of schooling were nine years. Most of the participants were currently out of work. Almost half of the women were injecting opiate users, while the remaining women were engaged with unsafe sex with others (Table 1).

Table 1. Sample Characteristics $(\mathrm{n}=47)$

\begin{tabular}{|c|c|}
\hline Characteristics & Values $^{\mathrm{a}}$ \\
\hline \multicolumn{2}{|l|}{ Participants } \\
\hline Injecting drug users & 24 \\
\hline Engagers with high-risk sex & 23 \\
\hline Median age, $y$ & 33 \\
\hline Age range, $y$ & $18-49$ \\
\hline Median years of schooling & 9 \\
\hline \multicolumn{2}{|l|}{ Living conditions } \\
\hline Unstable & 29 \\
\hline Living with family & 18 \\
\hline \multicolumn{2}{|l|}{ Marital status } \\
\hline Divorced & 29 \\
\hline Married & 9 \\
\hline Single/never married & 8 \\
\hline Widowed & 2 \\
\hline \multicolumn{2}{|l|}{ Employment } \\
\hline Out of work & 28 \\
\hline Home maker & 14 \\
\hline Employed & 3 \\
\hline Student & 2 \\
\hline \multicolumn{2}{|l|}{ Main drug of use } \\
\hline Opiates (e.g. opium or low purity heroin) & 26 \\
\hline Opiates (with impure methamphetamine) & 21 \\
\hline Initial age of drug use, $y$ & $18.9(5.2)$ \\
\hline Duration of drug dependence, $y$ & $10(8.1)$ \\
\hline Lifetime shared drug injection & 5 \\
\hline Currently living with injecting drug users & 2 \\
\hline
\end{tabular}

${ }^{\mathrm{a}}$ Values are expressed as mean, No., or No. (\%). 


\subsection{Barriers to Accessing and Adhering to HIV Services}

\subsubsection{Stigmatization}

An issue that impacted HIV service utilization was stigmatization at HIV centers. A theme that gradually emerged from the narratives was the notion of being stigmatized as "sex workers" while visiting mixed-gender HIV centers.

Women repeatedly mentioned that the close relationship between HIV infection and sex work created an environment where men patients and staff at HIV centers treated them unfairly. This concept was ultimately problematic because some women left the centers and did not receive HIV services.

Women internalized the stigma to the point where they felt that women-only centers were the only way that they could seek HIV services. The KI repeatedly stated that the emotional and personal lives of the women were negatively impacted through the internalization of the stigma surrounding HIV. Thirty-eight women and eight KI emphasized this problem. However, KI believed that stigma was part of the problem. Misconceptions about the future of HIV treatment among the women were also significant barriers to receiving HIV services.

A 38-year-old homeless woman who lived in a dormitory quoted as follow:

“... I'm upset. I need HIV counseling and maybe treatment. I and Nahid (a woman friend) didn't feel easy to go to an HIV center because people looked hard at us. Once I was there, they (male patients and doctors) viewed me as a bad woman (sex worker)... We need female-only centers for HIV treatment. Male doctors can't understand me because they aren't women..."

A registered HIV counselor who worked with at-risk women for more than ten years quoted as follow:

“... HIV services in Iran are male-dominated. The stigma is just the first reason to avoid these centers. Women with HIV service needs also don't enter or adhere to HIV services because they fear receiving a positive HIV status.... Feeling stigmatized exaggerates this issue. I suggest providing more nationwide education about the necessity and usefulness of HIV treatment...."

\subsubsection{A considerable Lack of Knowledge of Free HIV Centers in the Community}

A theme that gradually emerged from the interviews was a common misconception about the high cost of HIV services. KI stated that most drug treatment staff had the same misconception. A theme that repeatedly emerged from the narratives was that women did not care about HIV treatment before drug treatment entry. However, drug treatment entry provided an opportunity for them to consider HIV testing or treatment. Some women asked drug treatment staff about HIV centers in the community.

However, staff members were not able to provide adequate information about contact details and/or requisites for patient admission at HIV centers. While free HIV services are available in the Persian community, most women and drug treatment clinics did not have the most recent information about HIV services. Twenty-eight women and six KI emphasized this issue.

A 30-year-old woman who lived in a dormitory quoted as follow:

“... I thought it was just for some people, such as hospitalized HIV patients. I didn't know even sex workers could get free HIV treatment.... I didn't know it was free... My friend Maryam is HIV-positive. She used to inject heroin with a guy for ages. She was there once for testing and treatment. She told me to go..."

A doctor who worked with at-risk women at one of the study centers quoted as follow:

“...Drug centers just work on drug treatment. Their staff members don't consider the HIV problem to be part of their professional responsibilities. The contact details of HIV centers are easily available online... However, a few people know... The drug treatment staff should be officially educated about this issue..."

\subsubsection{Previous Traumatic Events}

Previous traumatic events, such as sexual or physical abuse, forced injection and violent attacks commonly emerged from the narratives. Most of the women had no actual knowledge that HIV service providers were professional medical teams. A mental generalization between previous traumatic events and HIV service providers was gradually identified from the narratives.

Some women believed that HIV service providers were likely to be the same as the people who had cheated them in the past. The KI thought that these women lacked knowledge about HIV service providers, and the women's previous traumatic experiences only worsened this situation because most HIV service providers were male and most women's previous traumatic events had occurred in their relationships with men. Twenty-one women and five KI identified previous traumatic events as strong barriers to accessing and adhering to HIV services.

A 35-year-old homemaker quoted as follow:

“...In the past, my father and brother physically abused me. So I can't trust male doctors to discuss HIV. I'm scared of more abuse. I can't trust any man...”

The director of an HIV center who had more than 10 years of work experience quoted as follow: 
“...Some at-risk women are scared to come for HIV treatment because they have no knowledge about us...They should be informed that the medical teams who provide HIV treatment are completely different from the people who have abused them in the past...We have published some booklets, brochures, posters, and handouts about the HIV centers. They should know that these centers are run by the government..."

\subsubsection{Misconceptions About the Quality of HIV Services}

Some women believed that the quality of the offered HIV services was poor. The women who were interviewed expressed a sense of reluctance in regard to discussing their HIV service needs with people at HIV centers because they believed that the services were not up-to-date.

Some of them held negative attitudes towards the quality of HIV services for a long time, and expressed similar themes of being reluctant in using these services. They had heard from other patients that HIV centers did not thoroughly met their HIV needs. Regarding the misconceptions associated with these services, it did not matter whether the women were infected and needed treatment; the concept of poor quality was still emotionally attached to HIV services.

Misconceptions played into the personal beliefs of the women in a difficult way. The quotes taken from the women illustrated various misconceptions that the women experienced, as they believed that the people at HIV centers were unable to support them in regard to their healthcare or medical needs. Twenty women and five KI emphasized this issue. However, the KI partly disagreed.

A 28-year-old HIV-positive sex worker who lived in a dormitory quoted as follow:

“...I don't know how they can understand my HIV problem and how they will treat me. I take methadone. What will happen to me if I take methadone with HIV medicines? I can't adhere to HIV services because I can't trust the quality of HIV services while taking methadone..."

A doctor who worked with at-risk women quoted as follow:

“...HIV centers have good medical and treatment facilities, but some at-risk women think the quality of these HIV services isn't good. This is because they have high expectations from HIV treatment. They expect to be treated in a short period of time. HIV centers are well-equipped... I believe health policy makers and drug clinics should provide at-risk women with more information about these services and the amount of support that HIV centers can provide..."

\subsubsection{A considerable Lack of a Supportive Referral System}

The themes that repeatedly emerged from the quotes showed that the women considered drug treatment cen- ters as the only reliable agents that were likely to refer them to HIV centers. However, drug treatment centers did not refer the women to receive HIV services. Some KI confirmed that there was no collaboration between drug treatment centers and HIV centers in regard to giving priorities to at-risk.

The poor supportive referral system was manifest in a number of interviews. Many women believed that patient admission at HIV centers required special requisites, and the drug treatment clinics were not informed about the admission requisites. This issue was identified by 20 women and five KI as a strong barrier to accessing and adhering to HIV services.

A 41-year-old homeless woman quoted as follow:

“...No one has referred me for HIV treatment. Drug clinics don't care about HIV. Once I went to an HIV center, I saw that they didn't care about sex workers...The drug clinic should give me a letter of referral for HIV treatment..."

A doctor who worked for more than seven years with at-risk women quoted as follow:

“...It isn't about money. There is no collaboration between drug and HIV treatment centers. Like public hospitals, HIV centers are busy with patients, so having a closely monitored collaborative relationship is hard. HIV centers should have a considerable priority in admitting at-risk women to drug treatment because of their high vulnerability to HIV and drug problems at the same time. At present, we don't have such a system in the country...Some laws should be approved for providing HIV and drug treatment in the same place..."

\section{Discussion}

As the most populated Persian Gulf country in Western Asia, in Iran, women drug users can easily attend gendermixed or women-only drug treatment centers for drug treatment (2). With the direct support of the government, free HIV treatment is widely available for both men and women in the community. Methadone maintenance treatment is also widely implemented to treat drug use. But, HIV service needs among women still need further professional consideration (2).

Unsafe sex and drug injection are common routes of HIV infection among at-risk women in Iran $(6,14,15)$. In summary, we found that the women had a need for receiving HIV services. However, some self-perceived internal and external barriers prevented them from accessing and adhering to the available services.

The women repeatedly raised the point that they experienced stigmatization because others believed that HIV was a social taboo in the community. This issue partly 
brought to light the negative image of women with HIV service needs due to perceptions regarding how they contracted it and how these negative images prevented them from receiving HIV services.

Studies in the US have found that women with HIV service needs are frequently stigmatized, and assumptions are made about their lives and the choices they have made (16). In New Zealand, a study found that HIV is constructed as a "men-related disease" (17).

Studies show that stigma is also a central issue in preventing people from being tested and treated for HIV in Iran (18). At-risk women in Iran may easily access community HIV centers; however, none of the centers have particularly targeted their needs or tailored specific interventions (14). Official reports in recent years have revealed that hard efforts have been undertaken by the government to eliminate stigma from HIV in Persian Iran (2). Nevertheless, more efforts are still required.

Persian women's misconceptions about the future of HIV treatment should be modified by providing ongoing education. Establishing female centers is suggested, but these centers should not segregate women clients. They may recruit only female staff in the hopes of creating nonjudgmental, women-only settings where women can feel comfortable and welcome to share difficult issues, such as sex work, drug problems, and HIV treatment. Further research is still required to better understand this emerging concept and to ultimately assess the feasibility and effectiveness of establishing female-only HIV centers.

Poor knowledge of the availability of free HIV serviceswas another barrierto receiving HIV services. A study in the US found that women generally reported poor knowledge among doctors regarding available antiretroviral medications in the community (19). Another study in the US indicated that limited knowledge of free HIV services in the community was a strong barrier to the implementation of successful antiviral therapy for HIV patients (20).

A study of at-risk women in Tehran found that some women had no knowledge about the address details or locations of HIV centers (2). Facilitating meaningful access to HIV services through increasing patient and staff knowledge is likely to encourage Persian women's access and adherence to these services. The provision of regular awareness workshops, training, and information updates at drug treatment centers is suggested. In addition, the massmedia in Iran, especially television, YouTube, and Facebook, should regularly inform people about the availability of free HIV services in the community. In public places, such as shopping malls, metro stations, hospitals, and bus stations, posters should be put up to inform people about the necessity of HIV testing and the availability of free HIV testing and treatment centers in the community.

Previous traumatic events were other self-perceived barriers among women that prevented them from seeking HIV treatment. However, KI partly attributed this issue to poor knowledge or high expectations about HIV treatment among women. Women were likely to expect that their previous traumatic events would berepeated in their relationships with HIV staff. Some sex workers in the US and Canada have reported avoiding care because of their previous traumatic events (21). At-risk women should be provided with adequate information about the safety of HIV centers. Staff members of the HIV centers in Iran should be trained to be more supportive towards at-risk women. Such women should be informed that HIV centers are governmental and the provided services are under the direct supervision of registered medical teams and government.

The study findings showed that misconceptions about the quality of HIV services were strong barriers against the utilization of HIV services. A study of 102 women in the US identified a common distrust of the health care system related to HIV (22). Further studies are needed to understand how trust in HIV service providers and the healthcare system is achieved and maintained, and also how trust is correlated with HIV-related health outcomes among atrisk women in the Persian community.

A poor supportive referral system was another identified barrier. Women were likely to be in a critical need for HIV counseling and treatment, but their drug treatment providers did not support them in accessing HIV services in the Persian community. A qualitative study of 21 healthcare providers and 5 patients found that an inadequate referral system, poor collaboration between different levels of the referral system, and insufficient knowledge about patient admission were strong barriers to healthcare access (23).

Most HIV prevention and care programs in Persian Iran do not reach at-risk women because their services have traditionally been designed for men (18). Any attempts to reach and work with at-risk women and to identify their HIV service needs are still unknown. Developing a supportive and integrated referral system has not been realized as a requirement to refer at-risk women who visit drug treatment centers to HIV centers. Persian health policymakers should contribute to forming this network by approving and implementing specific laws to this effect. Previous studies show that providing drug treatment and HIV services in one place is a must, but this issue has not been addressed across the country (2). The Persian ministry of health and medical education should consider this topic in the national strategic plans of the country for HIV testing and treatment in the near future. 


\subsection{Conclusions}

In this study, we identified some of the factors that were able to prevent at-risk women from accessing HIV services. There is a particular need for drug use treatment and HIV centers to address at-risk women' needs using a comprehensive and supportive approach. This method needs to include eliminating stigma and developing womansensitive HIV education and care materials for both women and staff.

\subsection{Limitations and Suggestions}

The study was limited to treatment seekers in Tehran. Therefore, the study findings may not be generalizable to other groups of at-risk women. The data were collected by self-reports, which are subject to underreporting or bias. Nevertheless, the collected data were anonymous and confidential. The sample was representative of the population that we studied.

As the first study in Western Asia, especially the Persian Gulf region, the qualitative methodology that we applied contributed to playing an important role in addressing the research gap. Studies show that qualitative research methods are more appropriate for investigating the sociocultural context of complex health behaviors (24). Further studies are suggested.

\section{Acknowledgments}

The authors would like to thank all the people who agreed for interviewing.

\section{Footnotes}

Authors' contribution: Mercedeh Samiei and Sepideh Aryanfard designed the study. Afsaneh Moradi and Roya Noori conducted the interviews. Hassan Rafiey and Hooman Naranjiha contributed to data analysis. All authors contributed to writing the paper, reading and approving the final draft of the paper.

Funding/Support: This work was approved and funded by the United Nations office on drugs and crime (UNODC) (Grant \#SSA/11/07).

\section{References}

1. Alavi SM, Behdad F. Seroprevalence study of hepatitis C and Hepatitis B virus among hospitalized intravenous drug users in Ahvaz, Iran (2002-2006). Hepat Mon. 2010;10(2):101-4. [PubMed: 22312381].

2. Iranian Ministry of Health and Medical Education . AIDS progress report.; 2014.

3. Stevens SJ, Tortu S, Coyle SL. Women drug users and HIV prevention: overview of findings and research needs. Binghamton: The Hawthorne Medical Press; 1998.
4. Weeks MR, Singer M, Himmelgreen DA, Richmond P, Grier M, Radda K. In: Women and substance abuse. Stevens SJ, Wexler HK, editors. New York: The Hawthorne Medical Press; 1998. Drug use patterns in substance abusing women: gender and ethnic differences in an AIDS prevention program.

5. Mirzazadeh A, Haghdoost AA, Nedjat S, Navadeh S, McFarland W, Mohammad K. Accuracy of HIV-related risk behaviors reported by female sex workers, Iran: a method to quantify measurement bias in marginalized populations. AIDS Behav. 2013;17(2):623-31. doi: 10.1007/s10461-012-0285-z. [PubMed: 22983500].

6. Alipour A, Haghdoost AA, Sajadi L, Zolala F. HIV prevalence and related risk behaviours among female partners of male injecting drugs users in Iran: results of a bio-behavioural survey, 2010. Sex Transm Infect. 2013;89 Suppl 3:iii41-4. doi: 10.1136/sextrans-2013-051201. [PubMed: 24064986].

7. Lotfi R, Tehrani FR, Yaghmaei F, Hajizadeh E. Barriers to condom use among women at risk of HIV/AIDS: a qualitative study from Iran. BMC women's health. 2012;12(1):13.

8. Kolahi AA, Rastegarpour A, Abadi AR, Nabavi M, Sayyarifard A, Sohrabi MR. The knowledge and attitudes of a female at-risk population towards the prevention of AIDS and sexually transmitted infections in Tehran.J Res Med Sci. 2011;16(11):1452-8. [PubMed: 22973347].

9. Razzaghi E, Nassirimanesh B, Afshar P, Ohiri K, Claeson M, Power R. HIV/AIDS harm reduction in Iran. Lancet. 2006;368(9534):434-5. doi: 10.1016/S0140-6736(06)69132-0. [PubMed: 16890814].

10. Day C, Nassirimanesh B, Shakeshaft A, Dolan K. Patterns of drug use among a sample of drug users and injecting drug users attending a General Practice in Iran. Harm Reduct J. 2006;3:2. doi: 10.1186/1477-75173-2. [PubMed: 16433914].

11. Pope C, Mays N. Reaching the parts other methods cannot reach: an introduction to qualitative methods in health and health services research. BMJ. 1995;311(6996):42-5. [PubMed: 7613329].

12. Corbin J, Strauss A. Basics of qualitative research: Grounded theory procedures and techniques. California: Sage Publications; 1990.

13. Daly KJ. Qualitative methods for family studies and human development. Sage Publications; 2007.

14. Khajehkazemi R, Osooli M, Sajadi L, Karamouzian M, Sedaghat A, Fahimfar N, et al. HIV prevalence and risk behaviours among people who inject drugs in Iran: the 2010 National Surveillance Survey. Sex Transm Infect. 2013;89 Suppl 3:iii29-32. doi: 10.1136/sextrans-2013051204. [PubMed: 24037249].

15. Nasirian M, Doroudi F, Gooya MM, Sedaghat A, Haghdoost AA. Modeling of human immunodeficiency virus modes of transmission in Iran.J Res Health Sci. 2012;12(2):81-7. [PubMed: 23241516].

16. Lawless S, Kippax S, Crawford J. Dirty, diseased and undeserving: the positioning of HIV positive women. Soc Sci Med. 1996;43(9):1371-7. [PubMed: 8913006].

17. Bennett J. New Zealand women living with HIV/AIDS: a feminist perspective. Nurs Prax N Z. 2007;23(1):4-16. [PubMed: 18044231].

18. Rahmati-Najarkolaei F, Niknami S, Aminshokravi F, Bazargan M, Ahmadi F, Hadjizadeh E, et al. Experiences of stigma in healthcare settings among adults living with HIV in the Islamic Republic of Iran.J Int AIDS Soc. 2010;13:27. doi: 10.1186/1758-2652-13-27. [PubMed: 20649967].

19. Boyer S, Marcellin F, Ongolo-Zogo P, Abega SC, Nantchouang R, Spire $B$, et al. Financial barriers to HIV treatment in Yaounde, Cameroon: first results of a national cross-sectional survey. Bull World Health Organ. 2009;87(4):279-87. [PubMed: 19551236].

20. Gahagan JC, Loppie CJ. Counting pills or counting on pills? What HIV+ women have to say about antiretroviral therapy. Can Woman Stud. 2001;21(2):118.

21. Sargeant $S$, Jones E. Barriers in access to primary health care for young HIV+ women: A qualitative research study. Vancouver: YouthCO AIDS Society and Positive Women's Network; 2008. 
22. Cunningham CO, Sohler NL, Korin L, Gao W, Anastos K. HIV status, trust in health care providers, and distrust in the health care system among Bronx women. AIDS Care. 2007;19(2):226-34. doi: 10.1080/09540120600774263. [PubMed:17364403].

23. Eskandari M, Abbaszadeh A, Borhani F. Barriers of referral system to health care provision in rural societies in iran. J Caring Sci. 2013;2(3):229-36. doi: 10.5681/jcs.2013.028. [PubMed: 25276731].

24. Corbin J, Strauss A. Basics of qualitative research: Techniques and procedures for developing grounded theory. Sage publications; 2014. 